Results The average monthly deliveries increased from 6.1 to 22.8 before and after the intervention respectively. The number of women attending for antenatal care increased from 11.5 to 33.6 per week before and after the intervention respectively.

Discussion Community health education empowers women, gives them a greater understanding of their health needs and has been successful at increasing the number of deliveries at Kabubbu HC. Community health education can be used alongside other interventions as a means to reduce maternal mortality.

\section{PL.59 FACTORS TO OVERCOME DELAYED DISCHARGE FOLLOWING CAESAREAN SECTION WITH AN ENHANCED RECOVERY PATHWAY}

doi:10.1136/archdischild-2013-303966.241

RR Duggal, S Walker, M Coker, M Dudley. Watford General Hospital, West Hertfordshire, UK

Enhanced recovery (ER) pathways improve clinical outcomes, the Department of Health $(\mathrm{DoH})$ suggesting several applications. Current published data within obstetrics is limited. Increased pressure on maternity beds; $39 \%$ of maternity units in England closing to admissions on one or more occasions in the year to 31 March 2007 [2], highlights a pivotal throughput parameter to benefit from an ER pathway.

Methods Survey of patients following elective caesarean section (CS) over a five-month period. Questionnaire designed against $\mathrm{DoH}$ guidelines [1].

Results 50 surveys completed.

$100 \%$ of patients were awake in recovery with regular analgesia prescribed. Mean pain score was $0.1 / 10$. $28 \%$ of respondents were nauseated or vomited in recovery. Mean time until discharge criteria were met was 153 minutes. Mean time for actual discharge from recovery was 213 minutes. $15 \%$ of patients were eating before discharge. $97 \%$ had a motor block on discharge. Urinary catheter remained in situ after 12 hours in $79 \%$ of patients. $100 \%$ of patients remained in hospital after 36 hours.

Discussion Patients delayed from returning to the ward may follow delayed discharges on the receiving ward, staff requirements to permit safe transfer and prolonged presence of an epidural (7\%) and urinary catheters (79\% after 12 hours). Promoting urinary catheter removal at 6 hours on a dedicated 'receiving bay' may reduce length of stay.

DoH suggests a change of culture may accompany discharges at 24 hours (providing all recovery factors have been cleared). We plan to implement a formal ER programme and re-audit in six months.

\section{PL.60 CONSEQUENCES OF FAILED INSTRUMENTAL DELIVERIES- HOW BAD IS IT?}

doi:10.1136/archdischild-2013-303966.242

'GL Stephen, ${ }^{2} \mathrm{R}$ Conroy, ${ }^{1} \mathrm{~S}$ Vause, ${ }^{1,2} \mathrm{C}$ Tower. ${ }^{1} \mathrm{Central}$ Manchester Foundation Trust, Manchester, UK; ${ }^{2}$ Maternal and Fetal Health Research Group, University of Manchester, Manchester, UK

Background Unsuccessful instrumental delivery (ID) is typically associated with increased risks of adverse outcomes. We aimed to investigate the fetal and maternal consequences of failed instrumental deliveries in our unit.

Methods Data was collected prospectively as part of ongoing audit of all IDs performed in our unit between October 2008 and March 2012.

Results Complete records were available for 2664 IDs. Delivery was unsuccessful in 75 (2.8\%). There was 1 maternal bladder injury and 1 hysterectomy. Unsuccessful ID was associated with increased blood loss (EBL), increased decision to delivery interval (DDI) and lower fetal Ph $(\mathrm{p}<0.001$ Mann-Whitney-U, table).

\section{Abstract PL.60 Table 1}

\begin{tabular}{lllll}
\hline Table & \multicolumn{2}{l}{ Unsuccessful } & \multicolumn{2}{l}{ Successful } \\
\hline & Median & Interquartile range (IOR) & Median & IOR \\
\hline Arterial Ph & 7.17 & $7.13-7.20$ & 7.21 & $7.16-7.26$ \\
Venous Ph & 7.22 & $7.17-7.26$ & 7.29 & $7.24-7.33$ \\
DDI (mins) & 47 & $32-62$ & 18 & $10-37$ \\
EBL (mls) & 650 & $500-900$ & 400 & $300-600$ \\
\hline
\end{tabular}

Sequential instruments were used in 189 (7\%), and were successful in $182 / 189$ cases $(96 \%)$. Compared with successful use, unsuccessful sequential instruments were associated with higher blood loss, increased DDI and lower fetal Ph ( $\mathrm{p} £ 0.06$, Mann Whitney-U). Two fetal injuries occurred where sequential instruments failed compared to 3 injuries out of 68 unsuccessful deliveries where only 1 instrument was used ( $p=0.07$ fishers exact test)

Conclusions ID was associated with a low risk of failure, but failure was associated with worse outcomes. Although sequential instrument use was associated with the poorest outcomes, outcomes were better if vaginal delivery was achieved in this situation. This highlights the need for careful and competent use of instruments in the presence of experienced operators.

\section{PL.61 PERIPARTUM HYSTERECTOMY - ANTICIPATED VERSUS UNANTICIPATED}

doi:10.1136/archdischild-2013-303966.243

N Maher, N Gleeson, T Darcy, B Byrne. Coombe Women and Infants University Hospital, Dublin 8, Ireland

Background Peripartum Hysterectomy $(\mathrm{PH})$ is a rare complication of pregnancy ${ }^{1}$. This study sought to determine the rate of $\mathrm{PH}$ in our population and compare aetiology and clinical outcomes in cases of $\mathrm{PH}$ that are anticipated and unanticipated.

Method Cases of PH for obstetric haemorrhage between 2006 and 2011 were identified from an $\mathrm{MOH}$ database. Data included maternal demographics, subspecialty of operator, aetiology for $\mathrm{PH}$, estimated blood loss(EBL), RCC units transfused, surgical complications and length of hospital stay.

Results 26 cases of PH (0.6/1000) were identified; 17 anticipated and 9 unanticipated. Placenta accreta (PA) was the indication for $\mathrm{PH}$ in 15/17 anticipated and 4/9 unanticipated cases. The remaining cases were attributed to uterine rupture (3/9), placenta previa, cervical ectopic and atony.

\section{Abstract PL.61 Table 1}

\begin{tabular}{llll}
\hline & Anticipated $\mathbf{n}=\mathbf{1 7}$ & Unanticipated $\mathbf{n}=\mathbf{9}$ & $\mathbf{p}$ value \\
\hline Mean Age (yrs) & $34+/-5$ & $36+/-3$ & \\
Mean Parity & 3 & 2 & \\
Mean EBL(L) & $5.5+/-3.6$ & $6.6+/-3.9$ & 0.89 \\
Range & $1.3-12.0$ & $2.8-14.3$ & \\
RCC units transfused & $7.2+/-5.2$ & $9.4+/-4.8$ & 0.4 \\
Intraoperative Complications & $41.1 \%$ & $33.3 \%$ & \\
Mean Operating time(mins) & $136+/-63$ & $190+/-95$ & 0.11 \\
Gynae Oncologist & $88 \%$ & $33 \%$ & \\
\hline
\end{tabular}

Discussion The majority of cases of $\mathrm{PH}$ are anticipated because of prenatal diagnosis of placenta accreta and are more likely to undergo elective delivery by a gynae-oncologist. Despite this EBL, RCC transfusion and postoperative complications are not significantly different from unanticipated cases of $\mathrm{PH}$, possibly reflecting the severity of cases diagnosed antenatally. Continuing research is required to improve the prenatal diagnosis of placenta accreta and to reduce perioperative blood loss with measures such as interventional radiology ${ }^{2}$. 


\section{REFERENCES}

1. Flood et al. Changing trends in peripartum hysterectomy over the last four decades. Am J Obstet Gynecol 2009 Jun; 200(6):632.e1-6.

2. Angstmann et al. Surgical management of placenta accreta: a cohort series and suggested approach. Am J Obstet Gynecol 2010 Jan; 202(1):38.e1-9. Epub 2009 Nov 17.

\section{PL.62 PERI-PARTUM HYSTERECTOMY: STILL REOUIRED FOR MANAGEMENT OF POST-PARTUM HAEMORRHAGE?}

doi:10.1136/archdischild-2013-303966.244

SM Barr, V Leburn, J Richmond. NHS Greater Glasgow \& Clyde, Glasgow, UK

Background Postpartum haemorrhage (PPH) complicates 5-10\% of all deliveries in the UK. While the use of uterotonic agents and compression devices has reduced the requirement for definitive surgical intervention, examination under anaesthetic, exploratory laparotomy and peri-partum hysterectomy remain in use to control intractable haemorrhage.

Method Cases of obstetric examination under anaesthetic $(n=66)$, exploratory laparotomy $(\mathrm{n}=13)$ and peri-partum hysterectomy $(\mathrm{n}=10)$ carried out in the Southern General Hospital were identified from theatre logs and the clinical risk reporting system from April 2009 to November 2012. Clinical features including estimated blood loss and interventional radiology involvement were extracted. Total births for this period was 16050 .

Findings The incidence of return to theatre for the management of $\mathrm{PPH}$ was $0.41 \%$ of total deliveries over this period. Of these cases, exploratory laparotomy was performed in $20 \%(n=13)$; peri-partum hysterectomy was required in $10.5 \%(n=7)$ of those cases, with an overall incidence of $0.06 \%$. A further three cases of caesarean hysterectomy were also identified; these were elective procedures for antenatally diagnosed invasive placentation. Where hysterectomy was performed, the underlying pathologies were uterine atony $(n=6)$ and invasive placentation (accreta and percreta, $n=4)$.

Conclusion Surgical intervention for management of severe postpartum haemorrhage is rarefor the overall obstetric population but remains necessary for management of uterine atony unresponsive to pharmacological management or compression and in cases of invasive placentation. Obstetric training should reflect this accordingly.

\section{PL.63 CAN WE SUSPECT SCAR DEHISCENCE OR RUPTURE AT EARLY STAGES?}

doi:10.1136/archdischild-2013-303966.245

S Bhaskar, W Forson. Royal Maternity Hospital, Belfast, UK

Introduction Uterine rupture is amongst the preventable obstetric complication that carries severe risks both to mother and baby

Aim Critically analyse the notes of women with rupture uterus over a period of 11 years and to reflect and learn from the outcome. Method and Settings Retrospective analysis of case notes of women with confirmed uterine rupture over a period of 11 years from January 2000-December 2011 at Royal Maternity Hospital, Belfast.

Results 17 women had confirmed uterine rupture in the study period of which 4 were preterm. All women except for one, had one or more term caesarean sections in past. The median interval between caesarean section and rupture was 3 years. Only $23 \%$ of women had induction of labour. Scar tenderness with or without suspicious CTG was the leading reason to suspect rupture. In nearly $70 \%$ of women maternal observations remained stable. Scar rupture was suspected only in $65 \%$ of women before the surgical intervention. 8 women (47\%) had either scar rupture or dehiscence of varying length while remaining $53 \%$ had extensions of scar rupture.
Conservative management remained the main stay of management. $35 \%$ of babies required neonatal care. The study also considered factors like uterine anomalies and surgeries, labour details including postpartum, staff involved etc.

Conclusion Trial of labour in previous section with successful outcome has long term implication on maternal health, while at the same time staff providing the care should be educated and trained to suspect the scar problems at early stages and intervene appropriately.

\section{PL.64 PYREXIA IN LABOUR: OUTCOME AND MANAGEMENT}

doi:10.1136/archdischild-2013-303966.246

S Elsayed, N Bozreiba, H Khan, S Cooley, S Coulter-Smith. Rotunda Hospital, Dublin, Ireland

Intrapartum fever can be due to an infectious or non-infectious aetiology and can lead to a variety of maternal and neonatal sequelae. Sepsis contributes significantly to maternal morbidity and mortality. Pyrexia may be the only symptom in early sepsis.

Aim To evaluate the incidence, management and outcome of intrapartum pyrexia in the Rotunda hospital, and to evaluate adherence to new hospital guidelines regarding septic screen and antibiotic therapy in intra-partum pyrexia.

Method Rretrospective audit of practise between 1 August and 30 September 2012. The presence or absence of maternal risk factors were reviewed and included: antenatal Group B Streptococcus (GBS), antenatal infections, preterm prelabour rupture of membranes (PPROM), prolonged rupture of membranes (ROM) and epidural analgesia. Intra-partum course parameters: management of pyrexia including resource to septic screen and intravenous antibiotics were evaluated.

Results 41 cases were selected and reviewed. The incidence of pyrexia in labour was $2.7 \%$ over the study period. The median maternal age was 29. The median gestational age at delivery was 40 . $80.5 \%$ were nulliparous. $5 \%$ had PPROM. $24 \%$ had prolonged spontaneous ROM (more than $18 \mathrm{hr}$ ). Only one woman was positive for GBS antenatally. 5 cases had GBS on HVS detected on septic screen and 1 case had GBS on placental swab. Labour was induced in $43.9 \% .27 \%$ delivered by emergency LSCS and 39\% by instrumental delivery.

Conclusion $88 \%$ of pyrexial women had a septic screen as per protocol. There was a poor culture lead from MSU with the highest yield from placental histology. Pyrexia in labour was associated with: Nulliparity, Induced labour, Prolonged ROM, Epidural analgesia and Operative delivery

\section{REFERENCE}

1. Maayan-Metzger A, Mazkereth R, Shani A, et al. Risk factors for maternal intrapartum fever and short-term neonatal outcome. Fetal Pediatr Pathol 2006;25:169.

\section{PL.65 DEFERRED CLAMPING OF THE UMBILICAL CORD: NEURAL PROGRAMMIING IN THE SURGEON AS A BARRIER TO CHANGE}

doi:10.1136/archdischild-2013-303966.247

1,2MM Beard. 'Wales Deanery, Cardiff, UK; ${ }^{2} A B M$ University Health Board, Swansea, UK

Recent UK and international guidelines have advocated deferred clamping of the umbilical cord. The evidence of benefit for the neonate is robust, particularly for preterm infants at high risk of anaemia, interventricular haemorrhage and necrotising enterocolitis. Midwifery colleagues use the practise routinely but obstetricians, involved mostly in operative and surgical deliveries, have inconsistently adopted deferred cord clamping.

Triggered by inconsistent takeup at audit, an online survey was circulated to Consultants and trainees in obstetrics and gynaecology 